\title{
Reusability of P3 facial filter in pandemic emergency: a 3D Analysis of a filter microstructure with X-Ray micro-tomography images after dry heat and UV sterilization procedures.
}

\author{
Luca Borro ( $\square$ luca.borro@opbg.net) \\ Bambino Gesù Children's Hospital - Rome \\ Massimiliano Raponi \\ Bambino Gesù Children's Hospital - Rome \\ Andrea Del Fattore \\ Bambino Gesù Children's Hospital - Rome
}

Franco Zanini

Elettra Sincrotrone - Trieste

Francesca Di Lillo

Elettra Sincrotrone - Trieste

Adriano Contillo

Elettra Sincrotrone - Trieste

Eleonora Di Piazza

Bambino Gesù Children's Hospital - Rome

Veronica Bordonaro

Bambino Gesù Children's Hospital - Rome

Alberto Eugenio Tozzi

Bambino Gesù Children's Hospital - Rome

Aurelio Secinaro

Bambino Gesù Children's Hospital - Rome

\section{Research Article}

Keywords: Covid19, Filtering Facepiece Respirator, Facial Protecion Mask, Reuse of facial mask

Posted Date: October 7th, 2020

DOI: https://doi.org/10.21203/rs.3.rs-89217/v1

License: (c) (1) This work is licensed under a Creative Commons Attribution 4.0 International License.

Read Full License 
Version of Record: A version of this preprint was published at International Journal of Environmental Research and Public Health on March 14th, 2022. See the published version at https://doi.org/10.3390/ijerph19063435. 
Reusability of P3 facial filter in pandemic emergency: a 3D Analysis of a filter microstructure with X-Ray micro-tomography images after dry heat and UV sterilization procedures.

P3 Facial Filters Reusability Analysis

Luca Borro ${ }^{1}$, Massimiliano Raponi ${ }^{2}$, Andrea Del Fattore ${ }^{3}$, Franco Zanini ${ }^{4}$, Francesca di Lillo ${ }^{4}$, Adriano Contillo $^{4}$, Eleonora Di Piazza ${ }^{5}$, Veronica Bordonaro ${ }^{1}$ Alberto E. Tozzi ${ }^{5}$, Aurelio Secinaro ${ }^{1}$

${ }^{1}$ Department of Imaging, Advanced Cardiovascular Imaging Unit, Bambino Gesù Children's Hospital, IRCCS, Rome, Italy;

${ }^{2}$ Health Directorate, Bambino Gesù Children's Hospital, IRCCS, Rome, Italy;

${ }^{3}$ Bone Physiopathology Research Unit, Genetics and Rare Diseases Research Area, Bambino Gesù Children's Hospital, IRCCS, Rome, Italy

${ }^{4}$ Elettra - Sincrotone Trieste, Trieste, Italy;

${ }^{5}$ Multifactorial and Complex Disease Research Area, Bambino Gesù Children's Hospital, IRCCS, Rome, Italy;

Word Count: 3.593

\section{Corresponding Author:}

\section{Dr. Luca Borro}

Department of Imaging

Bambino Gesù Children's Hospital, IRCCS

Piazza Sant'Onofrio 4 
00165 Rome, Italy

Phone: +39 06.6859.4815

Email: luca.borro@opbg.net 


\begin{abstract}
Objective: We sought to evaluate the effects of heating and UltraViolet (UV) sterilization on level 3 protection microstructure (P3) used on disposable filtering facepieces.
\end{abstract}

Intervention: P3 facial filter has been exposed to dry heat and UV sterilization procedures.

Methods: P3 facial filter samples underwent standardized sterilisation processes based on dry heat and UV irradiation techniques. We analysed key parameters of internal microstructure, such as fibres thickness and porosity, before and after sterilization using 3D data obtained with a synchrotron radiation based $\mathrm{X}$-ray computed microtomography (Micro-CT). The analyzed filter has two inner layers that we called "finer" and "coarser" layers. The "finer" layer consists of a dense fibers network while the "coarser" layer has a less compact fiber network. Results: Analysis on 3D images showed no statistically significant differences between P3 Filter of Controls and dry heat/UV sterilized samples. In particular, averages fibres thickness in the finer layer of Controls, $60^{\circ}$ dry heated and UV irradiated samples group was almost identical for each group. Average fibres thickness for coarser layer of Controls, $60^{\circ}$ dry heated and UV irradiated samples group was very similar measuring $19.33 \mu \mathrm{m}( \pm 0.47), 18.33 \mu \mathrm{m}( \pm 0.47)$ and $18.66 \mu \mathrm{m}( \pm 0.47)$, respectively. Of note, there was no substantial difference in fibres maximum thickness in the finer layers and coarser layers. For Controls group samples maximum thickness was on average of $11.43 \mu \mathrm{m}( \pm 1.24)$ in the finer layer and $59.33 \mu \mathrm{m}( \pm 6.79)$ in the coarser layer. Similarly, $60^{\circ}$ dry heated group samples were thickened $12.2 \mu \mathrm{m}( \pm 0.21)$ in the finer layer and $57.33 \mu \mathrm{m}( \pm 1.24)$ in the coarser layer, while UV irradiated samples group the mean max thickness is $12.23 \mu \mathrm{m}( \pm 0.90)$ in the finer layer and $58.00 \mu \mathrm{m}( \pm 6.68)$ in the coarser layer.

Theoretical porosity analysis resulted of $74 \%$ and $88 \%$, for finer and coarser layer, respectively. Theoretical porosity of finer layers tend to decrease on dry heat and UV irradiated samples when compared with the respective controls samples. 
Conclusions: Dry heat and UV sterilization processes do not substantially alter morphometry of the internal microstructure of the P3 filter samples studied with Micro-CT. The current study suggests that safe P3 filter facepiece reusability is theoretically feasible and should be further investigated.

\section{Keywords:}

Covid19, Filtering Facepiece Respirator, Facial Protecion Mask, Reuse of facial mask 


\section{Introduction}

In early 2020 the Coronavirus Pandemic involved the entire world, and the use of individual protective devices as protection from sars-Cov-2 infection is forcefully highlight. Sars-Cov-2 is a RNA virus first isolated in the Wuhan (China) in December 2019 and responsible for COVID-19 disease that causes severe interstitial pneumonia (1). The first meaningful way of diffusion of Sars-Cov-2 is through the saliva droplets with a diameters ranging from $1 \mu \mathrm{m}$ to $500 \mu \mathrm{m}$ (2) ejected from sneeze, cough and speech; another way of viral diffusion is by contact via contaminated surfaces (3); indeed, studies have shown that Sars-Cov-2 remains on contaminated surfaces for several hours, depending on the type of material is considered (4). The global pandemic has caused more than 6 million of cases worldwide, of which more than 388.759 have died as of June 2020 due to severe lung infections caused by pulmonary interstitial disease and lungs parenchymal consolidations.

The use of personal protective equipment is crucial to contain the spread of contagion worldwide in the absence of effective therapeutic approaches and vaccines (5). Previous works have highlighted the effectiveness of facial protection masks to protect against viral pathogens $(6,7)$ and, based on scientific evidence, governments around the world have strongly recommended the use of facial protection masks and respirators respectively for the general population and health professionals.

Different protective devices are used by the general population and in health contexts. These devices can be divided into different categories according to their function and material, including Cloth Masks, Surgical Masks and Filtering Facepiece Respirators.

The Filtering Facepiece Respirator (FFR) is high-protection face mask divided into different types depending on the design and the purpose of use. The nomenclature of FFR varies in different countries. FFP2 and FFP3 (UK designation) Respirators correspond to N95 and N99 (USA designation) respirators, respectively. In Europe, FFP respirators must meet the requirements of the 
European Standard EN149:2001 (4). The most frequently used FFR in hospitals are FFP2 (N95) and FFP3 (N99). The efficiency of FFP3 respirators is higher than FFP2 respirators, with a penetration of polydisperse $\mathrm{NaCl}$ aerosols particles through the filter $<1 \%$ for FFP2 and $<0.03 \%$ for FFP3 (8).

The FFRs consist of non-woven polypropylene fibrous filter media able to capture very small particles. There are several filtration mechanisms typical of facial respirators that characterize the interaction between the fibers of the porous media and the motion of the particles passing through the filter. These mechanisms are classified into four different types: interception, impaction, diffusion and electrostatic attraction (9).

The smaller particles (with a diameter of $<0.6 \mu \mathrm{m}$ ) that move at low speed within the filter are primarily blocked for impaction and interception. With the impaction, particles enter in the filter and are blocked by the impact with the fibers caused by the particle's inertia. In the interception, the particles passing close to the fiber following their streamline. Particles less than $0.1 \mu \mathrm{m}$ in diameter, on the other hand, are mainly captured by the diffusion mechanism which is caused by Brownian air motions that divert the motion of the particle until it comes into contact with a fiber. The electrostatic attraction instead requires that the fibers are electrically charged in order to determine an electrostatic attraction with the particles in motion (10).

The massive demand for masks from around the world affected by the Coronavirus Sars-Cov-2 pandemic has led to a sharp decrease in the supply of respirators on the market by industries. This causes an increased risk of contagion, especially for the health workers that are fighting the pandemic in intensive care units around the world.

For these reasons, it is relevant to understand the practical reusability of FFR to enable adequate protection from contagion diffusion to all health professionals and for the general population.

Recent studies have also found infecting viral particles on facial protective masks up to 4 days in the innermost layers and up to 7 days in the outermost layers (11). Several studies have confirmed 
that the Coronaviruses are stable at different temperatures: at 4 degrees Celsius $\left({ }^{\circ} \mathrm{C}\right)$, at room temperature $\left(20^{\circ} \mathrm{C}\right)$ and at $37^{\circ} \mathrm{C}$. However, these viruses become non-infectious after 90 minutes at $56^{\circ} \mathrm{C}, 60$ minutes at $67^{\circ} \mathrm{C}$ and 30 minutes at $75^{\circ} \mathrm{C}(12)$. It was also showed that $\mathrm{UV}$ radiation for 60 minutes decreases the viral load of the virus to negligible levels (11). There are few decontamination studies carried out using dry heat. In a recently published study, a filter sample was placed at $70^{\circ} \mathrm{C}$ in dry heat for $10,20,30$ and 60 minutes. These contamination cycles caused light drops in filtration performance; however, the reduction of filtration performance revealed with this method is lower than that observed by a $70 \%$ ethanol sterilization cycle leading to alterations of filter structure. The decontamination process carried out with UV rays, on the other hand, did not result in any significant structural alterations of the filter (13).

The studies analyzed so far have used highly professional sterilization systems and it would be particularly challenging to use them in the emergency context due to the Sars-Cov-2 pandemic, which would require continuous reuse of respirators otherwise not commercially available. These studies also focused more attention on UV decontamination and less on dry heat sterilization. Despite some reports showing a decrease of filtering capacity following filter sterilization treatments, none of the studies conducted a morphometric analysis of the micro-architecture of facial respiratory fibers to check the integrity of polypropylene fibers before and post decontaminating treatment.

This study evaluated the microstructure of the fibrous porous medium of a P3 filter sample (model 5935, 3M) before and after the filter sterilization procedure via dry heat and UV radiation. The aim of the study is to test whether the sterilization methods used cause alterations in the structure of the polypropylene filter fibers.

\section{Methods}

Samples 
Nine P3 Particulate Filters (mod. 5935 - 3M, St Paul) were used. This filter is commonly used as protection against particulates, dust, fumes and especially against spray droplets and it is compliant with AS/NZS 1716:2012 regulations. Given the scarcity of facemasks at a time of Coronavirus pandemic with global health crisis, we decided to use only one filter for our study from which we made subsamples in order to conduct our experiment without depriving the market of important protection devices especially for health workers.

The dimensions of entire filter are $105 \mathrm{~mm}$ (Height) x $85 \mathrm{~mm}$ (Max Width) x $\sim 4 \mathrm{~mm}$ (Thickness). The dimensions of our filter samples are: $10 \times 10 \mathrm{~mm}$.

\section{Sterilization}

Heat sterilization was performed incubating the samples at $60^{\circ} \mathrm{C}$ for 40 minutes using the Medite ${ }^{\circledR}$ TDO Sahara oven (Medite). Regarding the UV irradiation, samples were subjected to UV for 120 minutes using the G15T8 lamp (Sankio Denki).

\section{Synchrotron radiation X-RayMicrotomography}

The synchrotron radiation based X-ray computed microtomography was perfomed at the SYRMEP beamline of the Italian Synchrotron Radiation facility (Elettra, Trieste) (14) with a polichromatic Xray beam (mean energy of about $17 \mathrm{keV}$ ) and a sCMOS detector (Orca Flash, Hamamtsu) optically coupled with a 17- $\mu \mathrm{m}$ thick GGG (Gd3Ga5O12:Eu) scintillator. For each sample, 1800 tomographic projections were acquired over 180 degrees, with a single exposure time of $50 \mathrm{~ms}$, in propagationbased phase-contrast imaging mode (15). A phase retrieval algorithm à la Paganin (16) was applied prior to the tomographic reconstruction, performed through GPU-based filtered back-projection using the SyrmepTomoProject software (17). The reconstructed pixel size was 2 x 2 x $2 \mu \mathrm{m}^{3}$.

\section{Image Processing}


All image analyses were conducted using Fiji software. Fiji is an updated version of ImageJ, an OpenSource image processing program designed for multidimensional scientific images. It is highly extensible, with several plugins and scripts for performing a wide variety of tasks and parameter measurements on $2 \mathrm{D}$ or $3 \mathrm{D}$ images. After an initial analysis of 3D volumes from the X-Ray Microtomography of filter, it was immediately evident that the micro-architecture of the filter's polypropylene fibers could be defined as "bone-like" structure, consisting mainly of random-oriented rods in space and aimed at creating volume gaps within which the air flows. For this reason, BoneJ, a free open source ImageJ plugin often used for bone analysis, was used.

In order to deeply investigate the microstructure of sample fibers, an in-depth analysis was conducted using the Thickness (18) and Volume Fraction tools of Fiji BoneJ plugin.

The thickness tool allows to detect the mean thickness of the fibers in a fibrous porous media like a facepiece respirator filter while the Volume Fraction allows to analyze the ratio between void space and filled space occupied by fibers in the filter.

\section{Local Thickness Measurement}

The local thickness of a 3D geometry is calculated as the larger diameter of the sphere that fits within the structure at the point considered and that contains the point itself (Figure 1). Given a domain and a generic point in the structure, the local thickness calculated as the diameter of the largest sphere that contains the point nd that is wholly contained within the structure we want to measure:

$$
\tau(p)=2 \cdot \max (\{\mathbf{r} \mid \mathbf{p} \in \operatorname{sph}(\mathbf{x}, \mathbf{r}) \subseteq \Omega, x \in \Omega\})
$$

Where $\operatorname{sph}(\mathrm{x}, \mathrm{r})$ is the points inside the sphere with center $\mathrm{x}$ and radius $\mathrm{r}$. Since local thickness 
be defined for all points contained within the considered structure, it is necessary to define volume-based local thickness on the entire structure through an integration:

$$
\begin{gathered}
\overline{\mathbf{\tau}}=\frac{1}{\operatorname{Vol}(\Omega)} \iiint_{\Omega} \tau(\mathbf{x}) \mathbf{d}^{3} \mathbf{x} \\
\operatorname{Vol}(\Omega)=\iiint_{\Omega} \mathbf{d}^{3} \mathbf{x}
\end{gathered}
$$

The maximum local thickness is represented by the largest sphere that can be contained within the structure:

$$
\boldsymbol{\tau}_{\max }=\max (\{\boldsymbol{\tau}(\mathbf{p}) \mid \mathbf{p} \in \boldsymbol{\Omega}\})
$$

The thickness tool requires an 8 bit binarized images that can be obtained with the semiautomatic segmentation based on a thresholding algorithm in Fiji software. For the finer layer, segmentation was done with thresholding algorithm in "mean" mode. For the coarser layer, however, thresholding in the mode was used. The mean modality uses the mean of grey levels as the threshold cutoff. The default modality is a variation of IsoData mode (19) that divides the image in an object and background on the base of an initial threshold. Then all the averages of the pixels at or below the initial threshold and the pixels above are computed. The averages of those two values are computed and the process is repeated until the threshold is larger than the composite average.

\section{Theoretical Filter Porosity}

The theoretical filter porosity is the measure of the voids inside the porous media; it is defined as the ratio of void volume to the total volume of sample and is expressed as a percentage. Porosity is defined as the ratio: 


$$
\phi=\frac{\mathrm{v}_{\mathrm{v}}}{\mathrm{v}_{\mathrm{t}}}
$$

Where $\mathrm{Vv}$ is the volume of void space and $\mathrm{Vt}$ is the total volume of the considered material. For the facemask filters, the voids in the porous media contain air.

One of the methods for calculating the porosity of the material is the x-ray microtomography that allows to 3D reconstruct both the void space and the full spaces of the sample.

The porosity analysis is based on each of the nine samples analyzed through the Fiji software using BoneJ's Volume Fraction function.

\section{Statistics}

Statistical analysis was performed by one-way analysis of variance. Results were analyzed using the GraphPad Prism software version number 5 (San Diego, California, USA) and they were expressed as mean \pm standard deviation (SD). A result with $\mathrm{p}<0.05$ was considered statistically significant.

\section{Results}

The internal structure of the P3 filter $(5935,3 \mathrm{M})$ consists of two main layers: a finer layer and a coarser layer with $1.72 \mathrm{~mm}$ and $1.18 \mathrm{~mm}$ of thickness, respectively. The difference between these two layers is that the finer layer consists of more compact and "rodlike" fibers with a roughly cylindrical structure, while the coarser layer has thinning "plate-like" fibers with narrow rectangular section (Figure 2).

In order to avoid a data alteration of the thickness measurements due to the inhomogeneity of the layers, we analyzed separately the finer and the coarser texture of layers. The fibrous micro- 
architecture of the samples was evaluated by several image analysis techniques. The parameters chosen for the structural characterization of the filters are thickness and porosity of the nano-fibers that allow to quantify alterations of the fibrous microstructure in the filter samples following the sterilization processes.

In Figure 3, three-dimensional reconstruction of one sample volume is shown (Figure 3). The model was reconstructed with Mimics software (Materialise, Belgium) and allowed us to analyze the qualitative geometrical differences between finer and coarser layers.

\section{Thickness of nano-fibers}

The microtomography datasets for each sample were analyzed with BoneJ Thickness tool to determine the mean thickness of the polypropylene nano-fibers. The colorimetric map of the fiber thickness did not show any alterations of the dimensions of fibers in the three experimental groups (Figure 4).

The analysis of images confirmed that the sterilization procedure conducted on the samples did not result in any significant changes in the thickness size of the fibers, also measuring the two layers of the filters (finer and coarser layers) (Figure 5A-B). Moreover, Heat and UV treatments did not alter the average maximum fibers thickness in the layers (Figure 5C-D).

The analysis of the theoretical porosity of the filter samples analyzed showed no statistically significant differences in the microstructure of the polypropylene fibers after sterilization treatments (Figure 5E-F).

\section{Discussion}

The current study shows that the exposure of P3 facial filters to a sterilization process with dry heat $60^{\circ} \mathrm{C}$ and UVC irradiation does not result in substantial alterations to the fibers filter microstructure. 
FFR mask reusability during a pandemic has been investigated by scientific studies over the years. Several sterilization methods have been evaluated, such as UV radiation, sterilization in ethylene oxide and vaporized hydrogen peroxide as well as microwave oven irradiation and bleach (20). In this study exposure to microwave oven irradiation resulted in the fusion of two used respiratory samples while UV radiation with a 40W UV-C Light lamp for 5 minutes, ethylene oxide sterilization at $55^{\circ} \mathrm{C}$ for 1 hour and Vaporized Hydrogen oxide for 55 minutes proved to be the most effective sterilization methods.

Experimental results related to UV sterilization with a radiant dose of $120-950 \mathrm{~J} / \mathrm{cm}^{2}$ confirmed that this method can lead to an increase of particle penetration (up to 1.25\%) and a significant effect on the strength of the facial respirator material. At higher doses, the hardness of the respirator's fibrous layers suffers a significant loss of resistance, in some cases of more than $90 \%$ (21).

Different filter treatments as microwave-generated steam, warm moist heat and ultraviolet germicidal irradiation at $254 \mathrm{~nm}$ were able to determine a $>4-\log$ reduction of virus concentration in samples of facepiece respirators inoculated with $\mathrm{H} 1 \mathrm{~N} 1$ influenza virus. In $93 \%$ of cases, the virus was reduced to undetectable levels (13).

Based on the results obtained from previous works $(20,21)$ in which the sterilization processes led to an alteration of the filter structure and filtering capacity we decided to deepen this problem with an in-depth analysis of the relationship between sterilization process and structural integrity of facial protection filters.

In our study we technically analysed personal protective devices investigating potential for reusability, considering the scarcity of commercially available facial protection filters and that infecting viral particles on facial filters may be found for up to 7 days (13).

Differently from previous cited works that mainly used complex sterilization systems involving the use of ethylene oxide, vaporized perosside oxyde, moist heat and microwave we applied more accessible decontamination processes such as dry heat and UV rays. 
In order to conduct an initial technical assessment of device sterilization, we used the X-Ray Microtomography radiological imaging technique. Some other studies have already used X-Ray Micro-CT technology to study the porous filter microstructure (22) and the Scanning Electron Microscopy (SEM) (23).

In our study we decided to use the X-Ray Micro-CT because it allows to study entire volumes of material in three-dimensions with the possibility of making multiple measurements on different space planes.

This technique allowed us to analyse in detail with a resolution $2 \mu \mathrm{m}$ the fibrous structure of the filter and to better understand its geometry and morphometry details in order to detect any changes of the structure following the examined sterilization processes. From the analysis of the different filter samples, the sterilization processes did not statistically change the micro-architecture of the filters that showed no particular alterations of porosity compared to the average porosity values of similar filters reported in previous studies $(24,25)$. Similarly, the two planned sterilization processes did not result in a significant reduction in the average diameter of the fibers that make up the filter. As polypropylene is a material with a melting point around $160^{\circ} \mathrm{C}$, it is particularly resistant to lower temperatures even for extended periods of time $\left(60^{\circ} \mathrm{C}\right.$ for 40 minutes $)$ as demonstrated by this work.

\section{Limits of the study}

An important point to be considered to complete the sudy of filter's microstructure alterations after the sterilization process is the fibers electrostatic charge. In this study we did not evaluate if charge remained unchanged during and after the sterilization processes. Nevertheless, future investigation is needed to assess the permeability of post-sterilization filters with laboratory-specific tests that have not been the subject of this work.

This study also looked at a single type of P3 facial filter for facial semi-masks and not directly an FFP3 facial protection mask due to the scarcity of these devices at the moment of analysis. The number of samples analysed is also small. 
Although we have not detected significant alterations in the microstructure of the various samples analyzed, a large-scale study of samples could lead to different results.

Unlike the UV Rays, the $60^{\circ} \mathrm{C}$ dry heat sterilization process used in our study is not reported in literature as a widely used decontamination method mainly because it take longer time to get proper decontamination of the devices (26). Studies have also shown that the use of mere dry heat may not be enough to completely inactivate certain human viruses (27).

This is a limitation of this study that can be overcome in the future using a more conventional sterilization processes such as warm moist heat or microwave generated steam (28).

However, it was important to establish that the use of sterilization system that are not particularly complex and generally available within hospitals such as dry heat and UV irradiation have not resulted in substantial changes to the microstructure of the analyzed filters.

Based on the encouraging results obtained in this study, we believe that larger studies would be needed to better understand whether sterilization and reuse of facial protection filters can become a possible and standardized practice within hospitals during a pandemic.

\section{Acknowledgments}

No financial support was obtained for the current study.

\section{Declarations of interest}

All authors state that there is no conflict of interest and ethical adherence in this study.

\section{Authorship and manuscript preparation}

L. Borro: design of the work, writing the article, data interpretation and analysis 
M. Raponi: writing the article, data interpretation and analysis

A. Del Fattore: writing the article, experiments for sterilization of devices, data analysis

F. Zanini: micro-ct execution, image analysis reviewing

F. Di Lillo: micro-ct execution, writing the article

A. Contillo: Analysis Software and Computer Workstation setup, article reviewing

E. Di Piazza: writing the article

V. Bordonaro: article reviewing and images editing

A.E. Tozzi: literature review and article reviewing

A. Secinaro: micro-CT image analysis, writing the article

All coauthors approved the final manuscript and agree to be accountable for all aspects of the work in ensuring that questions related to the accuracy or integrity of any part of the work are appropriately investigated and resolved.

\section{Bibliography}

1. Xu X, Yu C, Qu J, Zhang L, Jiang S, Huang D, Chen B, Zhang Z, Guan W, Ling Z, et al. Imaging and clinical features of patients with 2019 novel coronavirus SARS-CoV-2. Eur J Nucl Med Mol Imaging (2020) 47:1275-1280. doi:10.1007/s00259-020-04735-9

2. Aliabadi AA, Rogak SN, Green SI, Bartlett KH. CFD simulation of human coughs and sneezes: A study in droplet dispersion, heat, and mass transfer. ASME Int Mech Eng Congr Expo Proc (2010) 7:1051-1060. doi:10.1115/IMECE2010-37331

3. Wei WE, Li Z, Chiew CJ, Yong SE, Toh MP, Lee VJ. Presymptomatic Transmission of SARS-CoV-2-Singapore. Morb Mortal Wkly Rep (2020) 69:411-415. 
4. Chin AWH, Chu JTS, Perera MRA, Hui KPY, Yen H-L, Chan MCW, Peiris M, Poon LLM. Stability of SARS-CoV-2 in different environmental conditions. The Lancet Microbe (2020) 5247:2004973. doi:10.1016/s2666-5247(20)30003-3

5. MacIntyre CR, Chughtai AA. Facemasks for the prevention of infection in healthcare and community settings. BMJ (2015) 350:1-12. doi:10.1136/bmj.h694

6. Maclntyre CR, Cauchemez S, Dwyer DE, Seale H, Cheung P, Browne G, Fasher M, Wood J, Gao Z, Booy R, et al. Face mask use and control of respiratory virus transmission in households. Emerg Infect Dis (2009) 15:233-241. doi:10.3201/eid1502.081167

7. Leung NHL, Chu DKW, Shiu EYC, Chan K-H, McDevitt JJ, Hau BJP, Yen H-L, Li Y, Ip DKM, Peiris JSM, et al. Respiratory virus shedding in exhaled breath and efficacy of face masks. Nat Med (2020) doi:10.1038/s41591-020-0843-2

8. Rengasamy S, Eimer BC, Shaffer RE. Comparison of nanoparticle filtration performance of NIOSH-approved and CE-marked particulate filtering facepiece respirators. Ann Occup Hyg (2009) doi:10.1093/annhyg/men086

9. Bulejko P. Numerical comparison of prediction models for aerosol filtration efficiency applied on a hollow-fiber membrane pore structure. Nanomaterials (2018) 8: doi:10.3390/nano8060447

10. Homaeigohar S, Elbahri M. Nanocomposite electrospun nanofiber membranes for environmental remediation. Materials (Basel) (2014) 7:1017-1045. doi:10.3390/ma7021017

11. Covid- RISS. Gruppo di lavoro ISS Biocidi COVID-19 e Gruppo di lavoro ISS Ambiente e Rifiuti COVID-19. (2020)

12. Duan SM, Zhao XS, Wen RF, Huang JJ, Pi GH, Zhang SX, Han J, Bi SL, Ruan L, Dong XP. Stability of SARS Coronavirus in Human Specimens and Environment and Its Sensitivity to Heating and UV Irradiation. Biomed Environ Sci (2003) 16:246-255. 
13. Fischer RJ, Morris DH, Sarchette S, Matson J, Bushmaker T, Yinda CK, Seifert SN, Gamble A, Williamson BN, Judson SD, et al. Assessment of N95 respirator decontamination and reuse for SARS-CoV-2. (2020)

14. Tromba G, Longo R, Abrami A, Arfelli F, Astolfo A, Bregant P, Brun F, Casarin K, Chenda V, Dreossi D, et al. The SYRMEP beamline of elettra: Clinical mammography and biomedical applications. AIP Conf Proc (2010) 1266:18-23. doi:10.1063/1.3478190

15. Rigon L. $x$-Ray Imaging with Coherent Sources. Elsevier B.V. (2014). doi:10.1016/b978-0444-53632-7.00209-4

16. Paganin D, Mayo SC, Gureyev TE, Miller PR, Wilkins SW. Simultaneous phase and amplitude extraction from a single defocused image of a homogeneous object. J Microsc (2002) 206:33-40. doi:10.1046/j.1365-2818.2002.01010.x

17. Brun F, Pacilè S, Accardo A, Kourousias G, Dreossi D, Mancini L, Tromba G, Pugliese R. Enhanced and Flexible Software Tools for X-ray Computed Tomography at the Italian Synchrotron Radiation Facility Elettra. Fundam Informaticae (2015) 141:233-243. doi:10.3233/FI-2015-1273

18. Dougherty R, Kunzelmann K-H. Computing Local Thickness of 3D Structures with ImageJ. Microsc Microanal (2007) 13:1678-1679. doi:10.1017/s1431927607074430

19. Ridler TW, Calvard S. Picture Thresholding Using. IEEE Trans Syst Man Cybern (1978) smc-8:630-632.

20. Viscusi DJ, Bergman MS, Eimer BC, Shaffer RE. Evaluation of five decontamination methods for filtering facepiece respirators. Ann Occup Hyg (2009) 53:815-827. doi:10.1093/annhyg/mep070

21. Lindsley WG, Martin SB, Thewlis RE, Sarkisian K, Nwoko JO, Mead KR, Noti JD. Effects of Ultraviolet Germicidal Irradiation (UVGI) on N95 Respirator Filtration Performance and 
Structural Integrity. J Occup Environ Hyg (2015) 12:509-517.

doi:10.1080/15459624.2015.1018518

22. Dalen G Van, Ginkel M Van, Hay G. 3D imaging and analysis of fibrous filter sheets using X-ray microtomography and image analysis . (1800)3-9.

23. Liu Z, Yu D, Ge Y, Wang L, Zhang J, Li H, Liu F, Zhai Z. Understanding the factors involved in determining the bioburdens of surgical masks. Ann Transl Med (2019) 7:754754. doi:10.21037/atm.2019.11.91

24. Polypropylene N, Lam T, Wu C, Huang S, Ko W, Huang Y. Multi-Scale Microstructure Investigation for a PM 2 . 5 Air-Filter E ffi ciency Study of.

25. Group K. The porosity of masks used in medicine. (2014)

26. Guideline for Disinfection and Sterilization in Healthcare Facilities (2008). Cent Dis Control Prev (2008)

27. Sauerbrei A, Wutzler P. Testing thermal resistance of viruses. Arch Virol (2009) 154:115119. doi:10.1007/s00705-008-0264-x

28. Yunoki M, Urayama T, Yamamoto I, Abe S, Ikuta K. Heat sensitivity of a SARS-associated coronavirus introduced into plasma products. Vox Sang (2004) 87:302-303. doi:10.1111/j.1423-0410.2004.00577.x 


\section{Figure Legends}

Figure 1. Schematic representation of the Local Thickness calculation method. The local thickness is calculated for each point $\mathrm{P}$ of a generic volume $\Omega$ as the diameter of the largest sphere inscribed in the volume and that containing the point considered.

Figure 2. Axial section of the P3 filter from X-Ray micro-CT images. A) Fine layer consisting of polypropylene fibers with a predominantly cylindrical section (rod-like). B) Coarser layer consisting of fibers with a thin predominantly rectangular section (plate-like) with a greater rarefaction of the fibers distribution.

Figure 3. 3D reconstruction of the Control Sample by a thresholding semi-automatic segmentation algorithm.

Figure 4. Colorimetric map of the fiber thickness in filter samples. The first two columns represent A-B) the Thickness of finer and coarser layers of Control Sample 1, C-D) Control Sample 2 and EF) Control Sample 3. In the second panel it is represented the thickness of finer and coarser layers of G-H) $60^{\circ} \mathrm{C}$ treated Sample 1 , I-L) $60^{\circ} \mathrm{C}$ treated Sample 2 and M-N) $60^{\circ} \mathrm{C}$ treated Sample 3. In the third panel it is represented the thickness of finer and coarser layers of O-P) UV irradiated Sample 1, Q-R) UV irradiated Sample 2 and S-T) UV irradiated Sample 3.

Figure 5. A-B) The finer and Coarser layers Thickness, C-D) Max Thickness and E-F) porosity for different types of samples. Results are expressed as mean \pm Standard Deviation. 
Figures

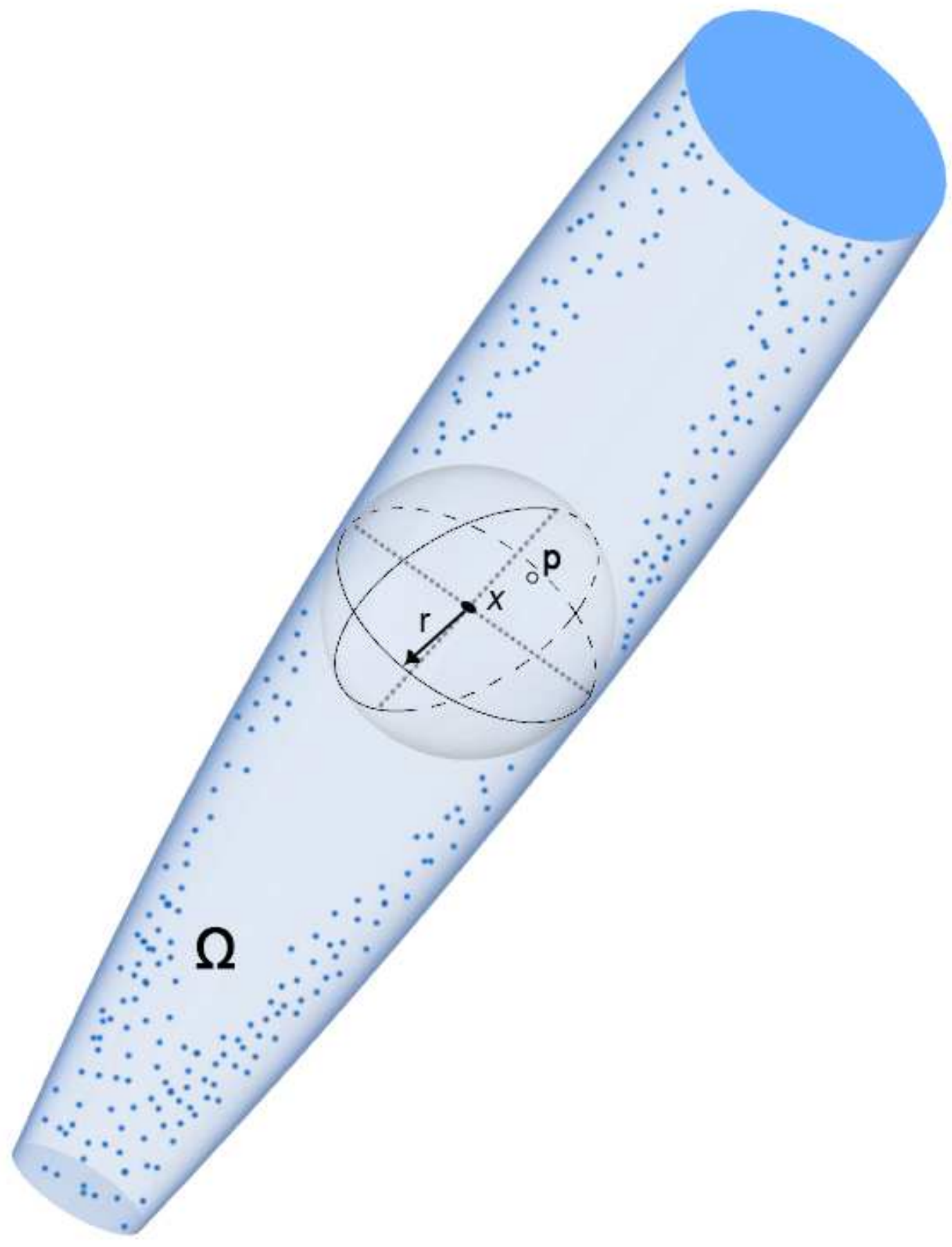

Figure 1

Schematic representation of the Local Thickness calculation method. The local thickness is calculated for each point $P$ of a generic volume $\Omega$ as the diameter of the largest sphere inscribed in the volume and that containing the point considered. 

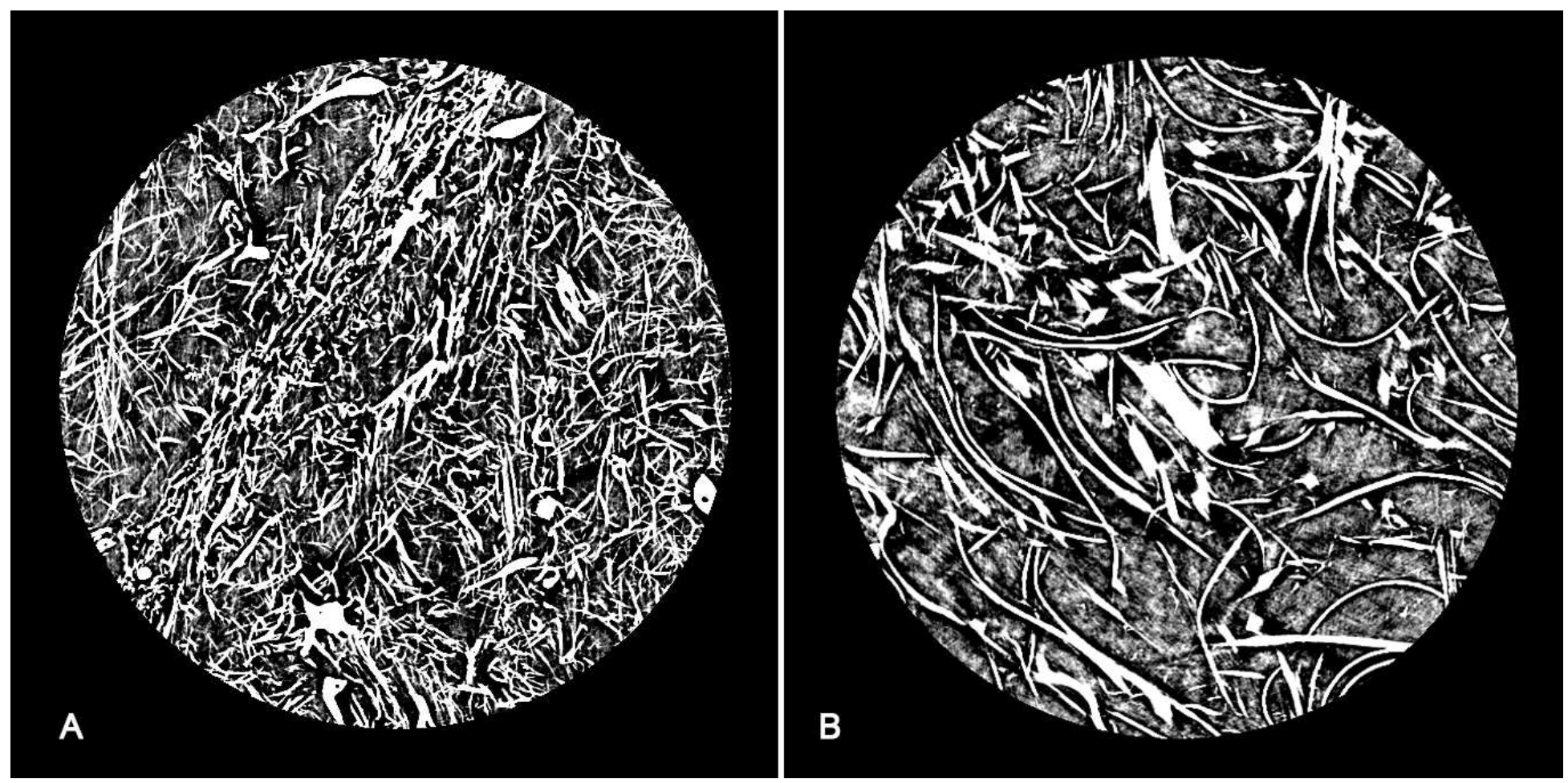

Figure 2

Axial section of the P3 filter from X-Ray micro-CT images. A) Fine layer consisting of polypropylene fibers with a predominantly cylindrical section (rod-like). B) Coarser layer consisting of fibers with a thin predominantly rectangular section (plate-like) with a greater rarefaction of the fibers distribution.
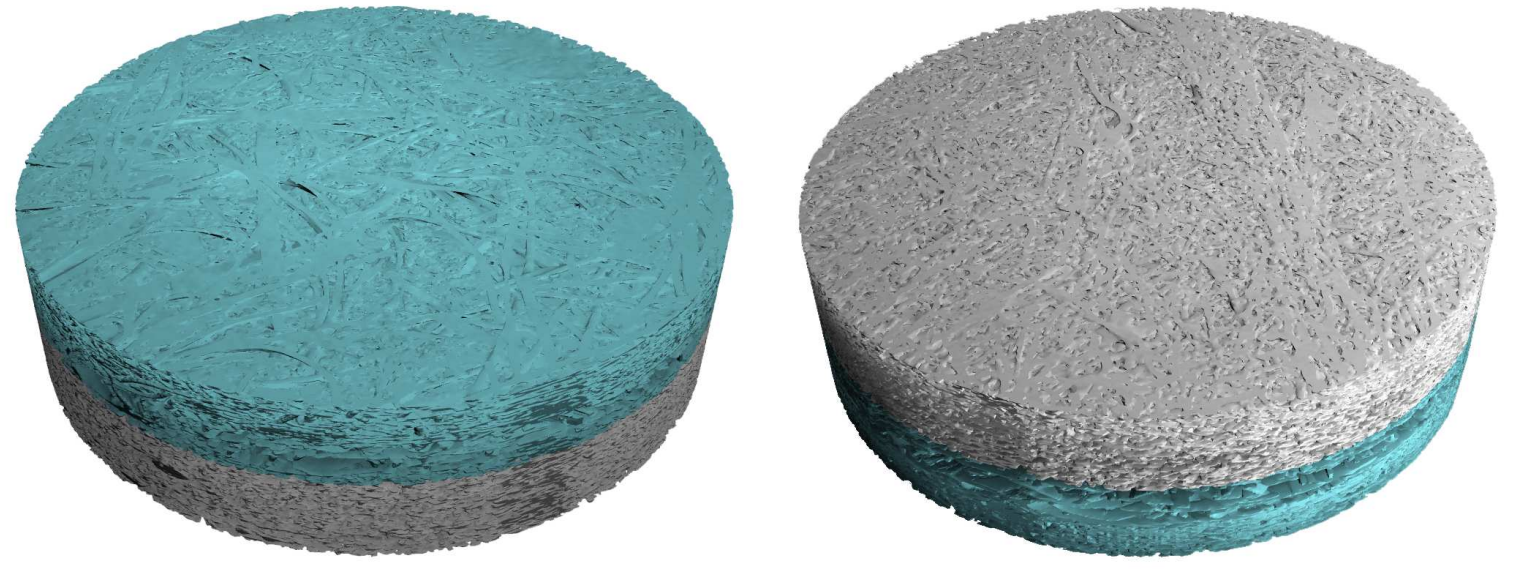

Figure 3

3D reconstruction of the Control Sample by a thresholding semi-automatic segmentation algorithm. 

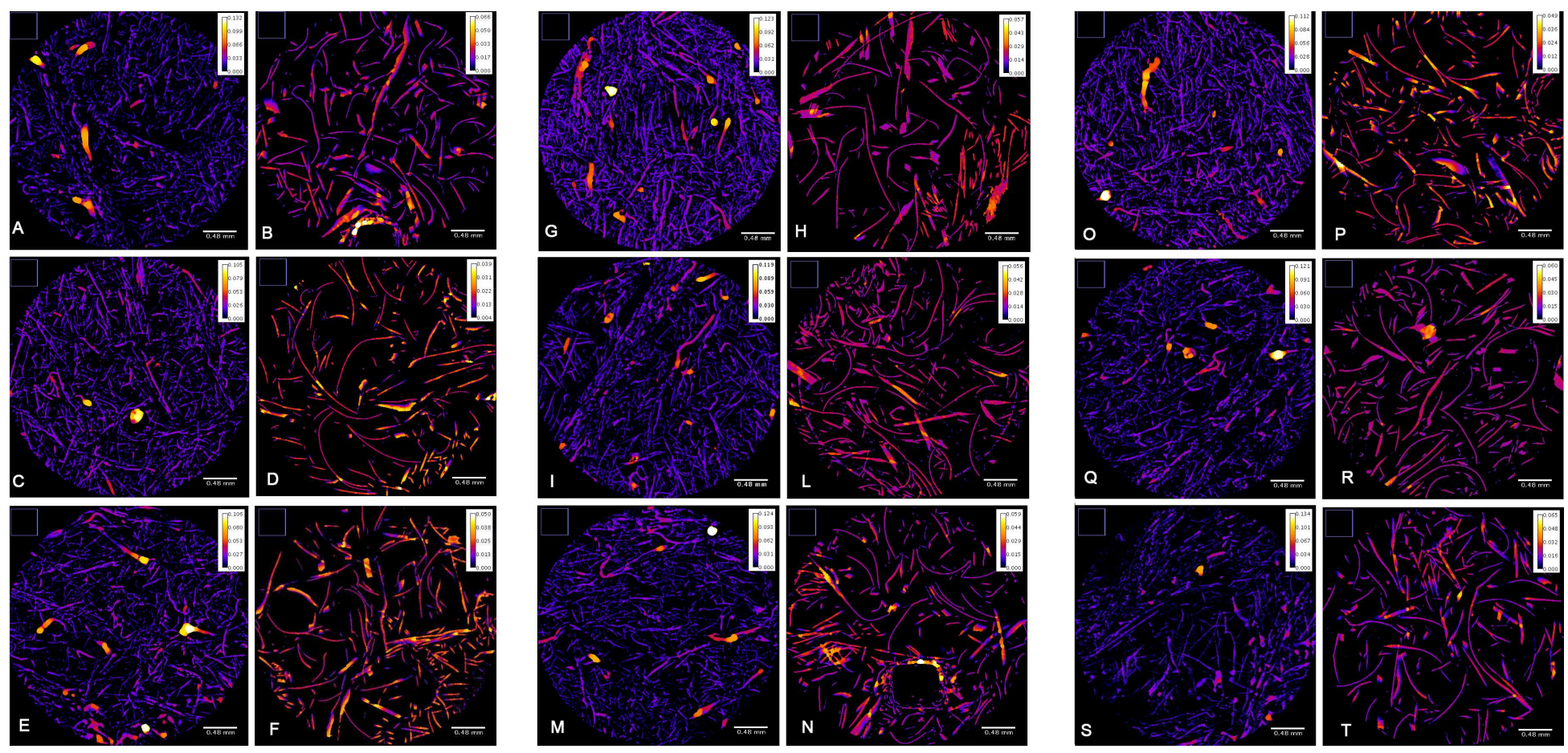

Figure 4

Colorimetric map of the fiber thickness in filter samples. The first two columns represent A-B) the Thickness of finer and coarser layers of Control Sample 1, C-D) Control Sample 2 and E-F) Control Sample 3. In the second panel it is represented the thickness of finer and coarser layers of G-H) $60^{\circ} \mathrm{C}$ treated Sample 1, I-L) $60^{\circ} \mathrm{C}$ treated Sample 2 and M-N) $60^{\circ} \mathrm{C}$ treated Sample 3. In the third panel it is represented the thickness of finer and coarser layers of O-P) UV irradiated Sample 1, Q-R) UV irradiated Sample 2 and S-T) UV irradiated Sample 3. 
A

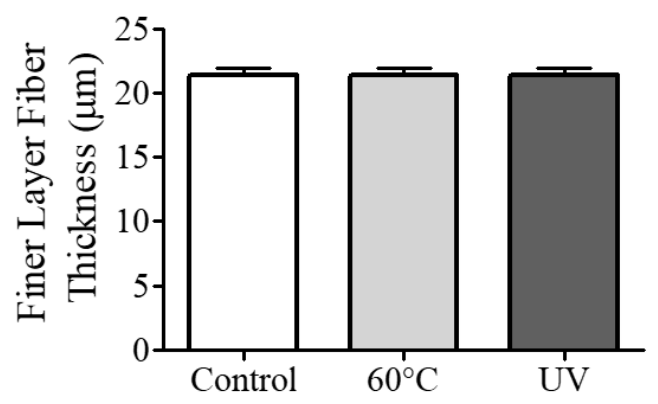

C

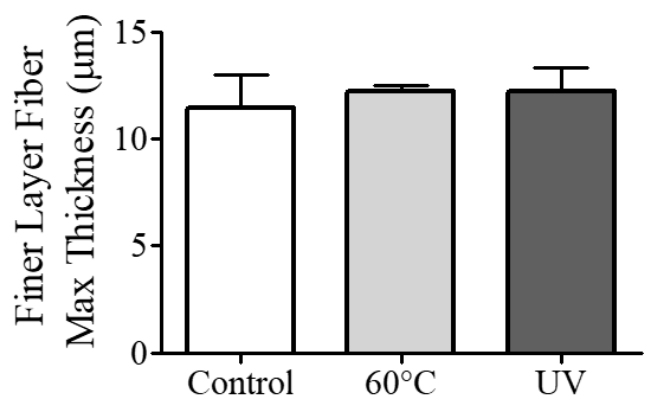

$\mathbf{E}$

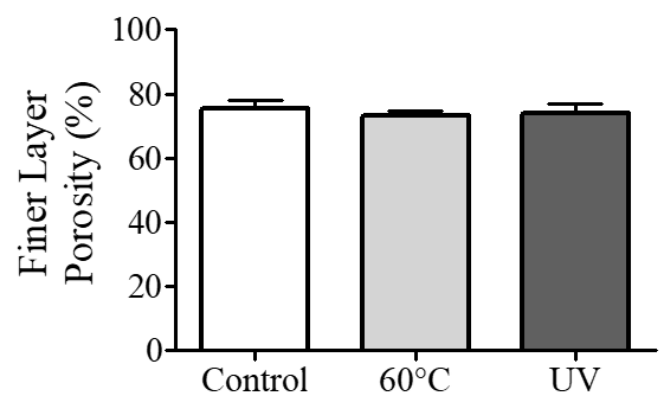

B

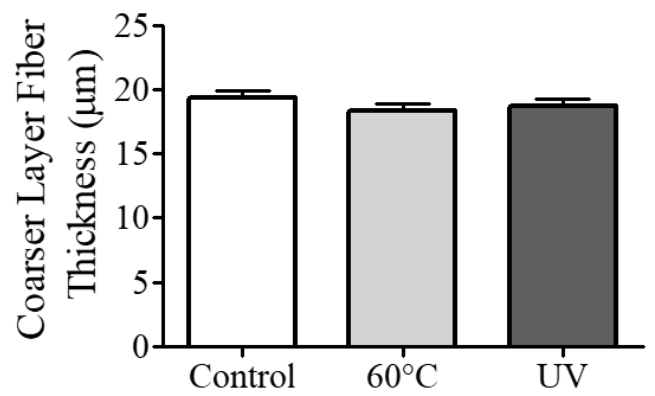

D

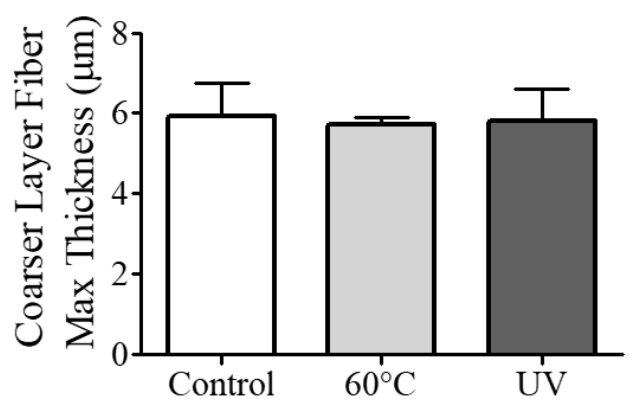

$\mathbf{F}$

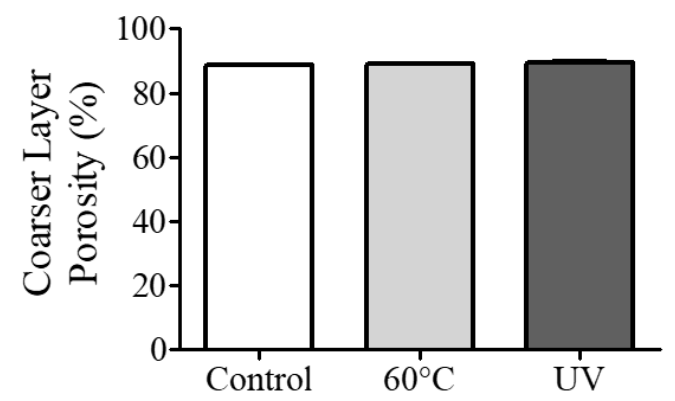

Figure 5

\section{Figure 5}

The finer and Coarser layers Thickness, C-D) Max Thickness and E-F) porosity for different types of samples. Results are expressed as mean \pm Standard Deviation. 